Homology, Homotopy and Applications, vol.12(2), 2010, pp.211-230

\title{
HOMOTOPY THEORY OF POSETS
}

\author{
GEORGE RAPTIS
}

(communicated by J. Daniel Christensen)

\begin{abstract}
This paper studies the category of posets $\mathcal{P}$ os as a model for the homotopy theory of spaces. We prove that: (i) Pos admits a (cofibrantly generated and proper) model structure and the inclusion functor $\mathcal{P}$ os $\hookrightarrow \mathcal{C}$ at into Thomason's model category is a right Quillen equivalence, and (ii) there is a proper class of different choices of cofibrations for a model structure on $\mathcal{P}$ os or $\mathcal{C}$ at where the weak equivalences are defined by the nerve functor. We also discuss the homotopy theory of posets from the viewpoint of Alexandroff $T_{0}$-spaces, and we apply a result of McCord to give a new proof of the classification theorems of Moerdijk and Weiss in the case of posets.
\end{abstract}

\section{Introduction}

This paper considers the category of posets from the viewpoint of homotopical algebra and shows that it provides a model for the homotopy theory of spaces. An elementary way to view the category of posets from a homotopical viewpoint is via the correspondence between posets and abstract simplicial complexes. The collection of chains in a poset defines an abstract simplicial complex and every weak homotopy type can be canonically represented this way. Conversely, for every geometric simplicial complex there is an associated poset of simplices that captures the homotopy type of the complex. In this paper, we show that this correspondence can be enriched in the following sense: the category of posets $\mathcal{P}$ os admits the structure of a model category that is Quillen equivalent with the model category of simplicial sets $\mathcal{S} \mathcal{S e t}$. The theory of model categories was introduced by Quillen $[\mathbf{2 1}$ and it has successfully established its importance in analysing homotopical phenomena. Modern treatments of the theory can be found in the recent monographs by Hirschhorn 11] and Hovey [12.

The category $\mathcal{P}_{o s}$ is a subcategory of the category of all small categories $\mathcal{C}$ at. Thomason $[\mathbf{2 8}$, showed that $\mathcal{C}$ at admits the structure of a model category where the weak equivalences are defined by the nerve functor $N: \mathcal{C} a t \rightarrow \mathcal{S} \mathcal{S}$ et. The nerve of a poset is precisely the simplicial set associated with the abstract simplicial complex of its chains. In Section 2, we show that Thomason's model category restricts to a

Received March 22, 2010, revised July 1, 2010; published on September 23, 2010.

2000 Mathematics Subject Classification: 18G55, 18B35, 55U35, 54G99.

Key words and phrases: model category, locally presentable category, poset, small category, Alexandroff space, classifying space.

This article is available at http://intlpress.com/HHA/v12/n2/a7

Copyright (C) 2010, International Press. Permission to copy for private use granted. 
(cofibrantly generated and proper) model structure on $\mathcal{P} o s$ and the inclusion functor $\mathcal{P}$ os $\hookrightarrow \mathcal{C}$ at is a right Quillen equivalence (see Theorem 2.6). In order to show this, we will first briefly recall the proof of Thomason's theorem to which our result is only an additional remark.

In Section 3, we study other model structures on $\mathcal{C}$ at and $\mathcal{P}$ os that have the same class of weak equivalences, but different cofibrations. More specifically, we apply results from the theory of combinatorial model categories $([\mathbf{3}, \mathbf{2 2}])$ to show that every adequate set of morphisms (see Definition 3.3) in $\mathcal{C}$ at or $\mathcal{P}$ os can be the generating set of cofibrations for a (cofibrantly generated and left proper) model structure on $\mathcal{C}$ at or $\mathcal{P}$ os (see Proposition 3.4). Furthermore, we show that there is a (cofibrantly generated and proper) model structure associated to every regular cardinal $\mu$, and it has the property that every $\mu$-presentable object is cofibrant (see Theorem 3.5).

A different link between posets and the homotopy theory of spaces is due to a theorem by McCord 18 (see Theorem 4.5). The category $\mathcal{P}$ os is well-known to be isomorphic with the category of topological spaces that are known as Alexandroff $T_{0}$-spaces (see Proposition 4.2). Under this correspondence, the subcategory of finite posets is isomorphic with the category of $T_{0}$-spaces with finitely many points. The homotopy theory of finite topological spaces has been studied by Stong [25. who reduced the classification of their homotopy types to a completely combinatorial description (see Theorem 4.12). McCord [18 showed that every geometric simplicial complex is canonically weakly homotopy equivalent with the Alexandroff $T_{0}$-space that is associated with the poset of its simplices. From this it follows that two posets are weakly equivalent if and only if the associated Alexandroff $T_{0}$-spaces are weakly homotopy equivalent. The theorems of Stong [25] and McCord [18 have been recently discussed in the survey articles by May [16, 17. Also, Barmak and Minian 2 introduced a notion of simple-homotopy for finite spaces that lies strictly between homotopy and weak homotopy and showed that it is a model for the simple-homotopy theory of simplicial complexes. In Section 4, we will discuss how the model structure on Pos can be interpreted in the category of Alexandroff $T_{0}$-spaces.

In Section 5, we discuss a few more ways that posets appear naturally in the homotopy theory of spaces. Since every CW-complex is homotopy equivalent to the classifying space of a poset, homotopy classes of maps $X \rightarrow Y$ between $\mathrm{CW}$-complexes can be represented by homotopy classes of maps $X \rightarrow B J$ for some poset $J$. In $[5.1$, we give a new proof of the classification theorems of $[\mathbf{2 0}]$ and $[\mathbf{2 9}]$ restricted to the case of posets. This says that homotopy classes of maps $X \rightarrow B J$ correspond to concordance classes of open coverings of $X$ well-indexed by $J$ (see Definition 5.1 ). In 5.2 , we discuss a useful well-known way to obtain a poset from an open covering of a space. In 5.3 , we remark that the cohomology of the topos of sheaves on a space is equivalent with the cohomology of the topos of presheaves on a poset.

\section{Notation}

We recall some standard notation. $\Delta$ will denote the category of finite ordinals $[n]=\{0<1<\cdots<n\}$ and order-preserving maps. $\Delta_{n}$ denotes the $n$-dimensional simplex, i.e., the functor $\Delta^{\mathrm{op}} \rightarrow \mathcal{S}$ et that is representable by $[n]$. Given a category $\mathcal{C}$ and objects $A$ and $B, \mathcal{C}(A, B)$ will denote the set of morphisms from $A$ to $B$. 
$\mathcal{T}$ op, $\mathcal{S}$ Set, $\mathcal{C}$ at and $\mathcal{P}$ os will denote the categories of topological spaces, simplicial sets, small categories and posets respectively. For every pair $(\mathcal{C}, \mathcal{W})$ of a category $\mathcal{C}$ together with a subcategory $\mathcal{W}$, the localisation of $\mathcal{C}$ at $\mathcal{W}$ will be denoted by $\mathcal{C}\left[\mathcal{W}^{-1}\right]$. If $\mathcal{C}$ is a model category, then we will write $\mathrm{Ho} \mathcal{C}$ to denote the homotopy category.

\section{Acknowledgements}

I would like to thank my D.Phil. supervisor Ulrike Tillmann for her support. I would also like to gratefully acknowledge the financial support of an EPSRC studentship and a scholarship from the Onassis Public Benefit Foundation.

\section{Thomason's model category}

The nerve functor $N: \mathcal{C} a t \rightarrow \mathcal{S S}$ et defines a class of weak equivalences in $\mathcal{C} a t$ which is of great interest in homotopy theory. We recall that the nerve $N C$ of a small category $C$ is a simplicial set whose $n$-simplices are the sequences of $n$ composable arrows in $C$; i.e.,

$$
N_{n} C=\mathcal{C} a t([n], C) .
$$

The face and degeneracy maps are given by the composition in $C$ and the insertion of identities respectively (or, in other words, by precomposition with the standard inclusion $\Delta \hookrightarrow \mathcal{C}$ at).

A morphism $f: C \rightarrow D$ in $\mathcal{C}$ at is called a weak equivalence if it induces a weak homotopy equivalence $N f: N C \rightarrow N D$ between the nerves of the categories. The class of weak equivalences in $\mathcal{C} a t$ will be denoted by $\mathcal{W}_{\mathcal{C} a t}$.

The nerve functor admits a left adjoint cat $: \mathcal{S S}$ et $\rightarrow \mathcal{C}$ at, called the categorical realisation, which takes a simplicial set $K$ to a category $\operatorname{cat}(K)$ with objects the 0 -simplices of $K$ and whose morphisms are freely generated by the 1-simplices of $K$ up to a "composition" relation for every 2 -simplex ${ }^{1}$. Alternatively, cat can be regarded as the cocontinuous extension of $\Delta \hookrightarrow \mathcal{C}$ at by the density of the Yoneda embedding $\Delta \hookrightarrow \mathcal{S S}$ et. Clearly, cat $N=1_{\mathcal{C} a t}$ and therefore $N$ embeds $\mathcal{C} a t$ in $\mathcal{S} \mathcal{S e t}$ fully faithfully as a reflective subcategory.

On the other hand, the unit transformation of the adjunction $1_{\mathcal{S S e t}} \rightarrow N$ cat is not even a weak homotopy equivalence in general and this can be testified by many non-trivial examples. For example, note that $\operatorname{cat}(K)$ is completely determined by the 2 -skeleton of $K$. However, the induced functor $\operatorname{Ho} N: \mathcal{C} a t\left[\mathcal{W}_{\mathcal{C} a t}^{-1}\right] \rightarrow \operatorname{Ho}(\mathcal{S} \mathcal{S e t})$ is an equivalence of categories. This can be shown by constructing a homotopy inverse to the nerve functor, that is: a functor $\Gamma: \mathcal{S S}$ et $\rightarrow \mathcal{C}$ at together with a natural weak homotopy equivalence $N \Gamma \rightarrow 1_{\mathcal{S S e t}}$; see [10]. An example of such a functor comes from the Grothendieck construction if we view a simplicial set $K$ as a simplicial category $K_{.}:[n] \mapsto K_{n}$ with values in discrete categories.

Thomason [28] showed that it is possible to lift the model structure on $\mathcal{S} S$ et to a model structure on $\mathcal{C}$ at via an adjoint pair of functors and that, in addition, the adjunction is a Quillen equivalence. The method of inducing model structures using

\footnotetext{
${ }^{1}$ The category $\operatorname{cat}(K)$ is also known as the fundamental category of $K$.
} 
pairs of adjoints is now a standard tool in the theory of model categories and it is described in the following proposition.

Proposition 2.1. Let $F: \mathcal{M} \rightleftarrows \mathcal{D}: G$ be an adjunction. Assume that $\mathcal{M}$ is a cofibrantly generated model category with $I$ and $J$ sets of generating cofibrations and trivial cofibrations respectively, and $\mathcal{D}$ is a category that admits all small limits and colimits. Suppose that the following are satisfied:

(a) the domains of FI (resp. FJ) are small relative to FI-cellular (resp. FJcellular) morphisms,

(b) $G$ takes $F J$-cellular morphisms to weak equivalences in $\mathcal{M}$.

Then there exists a cofibrantly generated model structure on $\mathcal{D}$, where

(i) $f$ in $\mathcal{D}$ is a weak equivalence (resp. fibration) if $G(f)$ is a weak equivalence (resp. fibration),

(ii) the class of cofibrations is the class of morphisms that have the left lifting property with respect to the trivial fibrations,

(iii) FI is a set of generating cofibrations and FJ is a set of generating trivial cofibrations.

Moreover, the adjunction becomes a Quillen adjunction of model categories.

Proof. See [11, Theorem 11.3.2].

Thomason 28 considered the adjunction

$$
\text { cat } S d^{2}: \mathcal{S S e t} \rightleftarrows \mathcal{C} a t: \operatorname{Ex}^{2} N
$$

where $S d: \mathcal{S S e t} \rightleftarrows \mathcal{S S}$ et $:$ Ex is an adjoint pair defined by Kan $1 \mathbf{1 3}$. We recall the definition of the subdivision functor $S d$. Firstly, $S d$ is defined on $\Delta$ as follows: for every $n \geqslant 0, S d \Delta_{n}$ is the nerve of the poset of the non-degenerate simplices of $\Delta_{n}$, i.e., the poset of non-empty subsets of $[n]=\{0<1<\cdots<n\}$. Then $S d$ : SSet $\rightarrow \mathcal{S S e t}$ is the Kan extension along the Yoneda embedding $\Delta \hookrightarrow \mathcal{S S e t}$; i.e.,

$$
S d K=\operatorname{colim}_{\Delta \downarrow K} S d \Delta_{n}
$$

for every simplicial set $K$. The elements of $(S d K)_{n}$ can be represented by equivalence classes of pairs $[x, \theta]$ where $x \in K_{m}$ and $\theta \in\left(S d \Delta_{m}\right)_{n}$. Moreover, there is a natural weak homotopy equivalence $S d K \rightarrow K$ for every simplicial set $K$. The extension functor Ex is defined to be the right adjoint of $S d$. Equivalently, the $n$-simplices of Ex $K$ correspond to simplicial maps $S d \Delta_{n} \rightarrow K$. It has the following important properties that we mention without proof (see $\mathbf{9}]$ ):

(a) Ex preserves Kan fibrations,

(b) there is a natural weak homotopy equivalence $X \rightarrow \operatorname{Ex} X$ (and hence $S d \dashv \operatorname{Ex}$ is a Quillen equivalence), and

(c) $\operatorname{Ex}^{\infty} K=\operatorname{colim}_{n} \operatorname{Ex}^{n} K$ is a Kan (or fibrant) simplicial set.

Property (b) implies that a morphism $f: C \rightarrow D$ is a weak equivalence if and only if $\operatorname{Ex}^{2} N f: \operatorname{Ex}^{2} N C \rightarrow \operatorname{Ex}^{2} N D$ is a weak homotopy equivalence in $\mathcal{S} S$ et. A morphism $f: C \rightarrow D$ is called a Thomason fibration if the simplicial map $\operatorname{Ex}^{2} N f$ is a Kan fibration, and a Thomason cofibration if it has the left lifting property with 
respect to all trivial Thomason fibrations, i.e., the Thomason fibrations which are also weak equivalences. Let $\mathrm{Cof}_{T}$ and $\mathrm{Fib}_{T}$ denote respectively the classes of Thomason cofibrations and fibrations in $\mathcal{C}$ at.

Theorem 2.2 (Thomason [28]). The classes weak equivalences $:=\mathcal{W}_{\mathcal{C} \text { at }}$, cofibrations $:=\mathrm{Cof}_{T}$ and fibrations $:=\mathrm{Fib}_{T}$ define a proper cofibrantly generated model structure on $\mathcal{C}$ at and the adjunction

$$
\text { cat } S d^{2}: \mathcal{S S e t} \rightleftarrows \mathcal{C} a t: \mathrm{Ex}^{2} N
$$

is a Quillen equivalence. Moreover, the sets

1. $\mathcal{I}=\left\{\operatorname{cat} S d^{2} \partial \Delta_{n} \rightarrow \operatorname{cat} S d^{2} \Delta_{n} \mid n \geqslant 0\right\}$, and

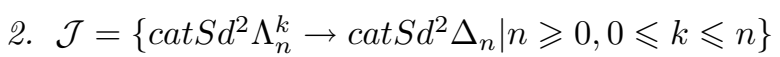

are sets of generating cofibrations and trivial cofibrations respectively.

Let us recall the main idea of Thomason's proof. The essential bulk of the proof reduces to proving the following claim, which amounts to the non-trivial part of condition (b) of Proposition 2.1; for every diagram

$$
B \stackrel{j}{\leftarrow} A \rightarrow C,
$$

where $j \in \mathcal{J}$, the canonical map $N C \rightarrow N\left(C \cup_{A} B\right)$ is a weak homotopy equivalence. This leads to the following definition of cofibrations in $\mathcal{C} a t$.

Definition 2.3. An inclusion $i: A \rightarrow B$ in $\mathcal{C}$ at is called a formal cofibration if for every diagram

$$
B \stackrel{i}{\leftarrow} A \rightarrow C
$$

the canonical map $N C \cup_{N A} N B \rightarrow N\left(C \cup_{A} B\right)$ is a weak homotopy equivalence.

For example, the unique morphism $\varnothing \rightarrow B$ is a formal cofibration for every small category $B$. The class of formal cofibrations singles out a property that every candidate class of morphisms needs to satisfy in order to qualify as a class of cofibrations for a left proper model structure on $\mathcal{C}$ at where $\mathcal{W}_{\mathcal{C} a t}$ is the class of weak equivalences. Thomason identified an auxiliary class of formal cofibrations called Dwyer morphisms. A Dwyer morphism is an inclusion of a sieve $i: A \rightarrow B$ such that there is a cosieve $W$ in $B$ containing $A$ and the inclusion $j: A \rightarrow W$ admits a right adjoint. Recall that an inclusion functor $i: A \rightarrow B$ is called a sieve if every morphism $b \rightarrow i(a)$ in $B$ is also in $A$. A cosieve is the dual object: $i: A \rightarrow B$ is a cosieve if $i^{\text {op }}: A^{\text {op }} \rightarrow B^{\text {op }}$ is a sieve. The class of Dwyer morphisms is closed under pushouts and transfinite compositions $[\mathbf{2 8}$, but not under retracts [6].

The concept of a Dwyer morphism corresponds intuitively to the topological notion of an inclusion of spaces being a neighborhood deformation retract, but it is too strong. A slightly weaker notion is needed and it was defined by Cisinski [6] $i: A \rightarrow B$ is a pseudo-Dwyer morphism if it is an inclusion of a sieve, and there is a cosieve $W$ in $B$ that contains $A$ and a retraction $r: W \rightarrow A$ together with a natural transformation $\eta: i r \rightarrow 1_{W}$ such that $\eta(a)=1_{a}$ for all $a \in \mathrm{ob} A$. 


\section{Proposition 2.4.}

(a) The class of Dwyer morphisms is closed under pushouts and transfinite compositions. The class of pseudo-Dwyer morphisms is closed under retracts, pushouts and transfinite compositions.

(b) For every monomorphism $K \hookrightarrow L$ in $\mathcal{S}$ Set, the morphism

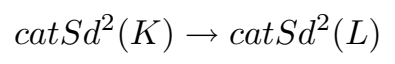

is a Dwyer morphism. In particular, every morphism in $\mathcal{I}$ and $\mathcal{J}$ is a Dwyer morphism.

(c) Every pseudo-Dwyer morphism is a formal cofibration. In particular, every morphism in $\mathcal{J}$ is a formal cofibration.

Proof. For (a), see [6] and [28. For (b), see [28, Proposition 4.2]. (c) is the main technical fact required for the proof of Theorem $[2.2$, and it is also of independent interest. A proof can be found in [28, Proposition 4.3] (see also [7, 5.2]).

Since every morphism in $\mathcal{I}$ is a Dwyer morphism, then Proposition 2.4(a) implies that every Thomason cofibration is a pseudo-Dwyer morphism. Proposition 2.4(b) reveals the point of considering the twofold composite $S d^{2}$ : if one considers (cat $S d$, $\operatorname{Ex} N)$ or $(c a t, N)$ instead, then property (b) of Proposition 2.1 is not true. In other words, a comparison between the homotopy theory of simplicial sets and the homotopy theory of small categories should pay special attention to the homotopic effect of taking pushouts in $\mathcal{C}$ at. The nerve functor does not preserve pushout diagrams, not even up to homotopy, even when the pushout is along a full inclusion (e.g., $\operatorname{NcatSd}\left(\Delta_{2} / \partial \Delta_{2}\right) \cong \Delta_{1}$, whereas $N \operatorname{cat} S d \Delta_{2} / N \operatorname{cat} S d \partial \Delta_{2} \simeq S^{2}$; see [10]). Thus it is necessary to find sufficient conditions for a pushout diagram in $\mathcal{C}$ at to be a homotopy pushout diagram in $\mathcal{S} S$ et. By Proposition 2.4(c), this happens in the presence of a pseudo-Dwyer morphism.

Let $\mathcal{P}$ os denote the full subcategory of $\mathcal{C}$ at whose objects are the partially ordered sets (posets). The full inclusion $i: \mathcal{P}$ os $\rightarrow \mathcal{C}$ at admits a left adjoint pos: $\mathcal{C} a t \rightarrow \mathcal{P}$ os called the posetal reflection. Given a small category $C$ and $a, b \in$ ob $C$, we write $a \leqslant b$ if there is a morphism $a \rightarrow b$ in $C$ and $a \sim b$ if both $a \geqslant b$ and $a \leqslant b$ hold. Then $\operatorname{pos}(C)$ is the partial order on the set of equivalence classes ob $C / \sim$ induced by $\leqslant$.

Lemma 2.5. The inclusion $i: \mathcal{P}$ os $\rightarrow \mathcal{C}$ at preserves directed colimits and pushouts along a Dwyer morphism.

Proof. This can be verified directly from the definition of colimits in $\mathcal{C}$ at. Alternatively, note that both $\mathcal{C}$ at and $\mathcal{P} o s$ are locally finitely presentable categories. A right adjoint between locally finitely presentable categories is an accessible functor, and it preserves directed colimits if and only if its left adjoint preserves finitely presentable objects [1, Theorem 1.66]. The functor pos: $\mathcal{C}$ at $\rightarrow \mathcal{P}$ os has this property because a finitely presentable small category has a finite set of objects. Dwyer morphisms mess with the order relation in a moderate way, so that the required pushouts are preserved. More precisely, it is useful to know explicitly how to construct the pushout 
in $\mathcal{P}$ os along a Dwyer morphism (or, more generally, along a sieve). Let

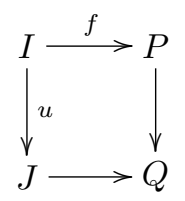

be a pushout square of posets in $\mathcal{C}$ at where $u$ is a sieve. Then $Q$ is naturally isomorphic with the poset whose underlying set is ob $P \sqcup(\mathrm{ob} J-\mathrm{ob} I)$, and the order is induced by the orders of $P$ and $J$ and a relation $j \geqslant f(i)$ for every $j \geqslant i$ in $J$.

It is natural to ask whether the adjunction pos: $\mathcal{C}$ at $\rightleftarrows \mathcal{P}$ os $: i$ induces a model structure on $\mathcal{P}$ os or, in other words, whether Thomason's model structure restricts to a model structure on $\mathcal{P}$ os. For every simplicial set $K$, the category $\operatorname{cat} S d^{2} K$ is a poset [28, Lemma 5.6]. When $K$ comes from an abstract simplicial complex, $c a t S d^{2} K$ is the poset of non-empty chains of simplices of $K$ ordered by inclusion. In particular, cat $S d^{2} \Delta_{n}$ is the poset of non-empty chains of subsets of $[n]=\{0<1<\cdots<n\}$. Therefore there is an adjunction $\operatorname{cat} S d^{2}: \mathcal{S} \mathcal{S}$ et $\rightleftarrows \mathcal{P}$ os $: \operatorname{Ex}^{2} N$.

Let us denote by $\mathcal{W}_{\mathcal{P}}$ os the class of weak equivalences in the category $\mathcal{P}_{\text {os }}$. We are going to use the same notation $\mathrm{Cof}_{T}$ and $\mathrm{Fib}_{T}$ to denote the classes of Thomason cofibrations and Thomason fibrations between posets. It will be clear what underlying category is considered each time. Note that the classes of Dwyer and pseudo-Dwyer morphisms coincide in $\mathcal{P}$ os.

Theorem 2.6. The classes weak equivalences $:=\mathcal{W}_{\mathcal{P}_{o s}}$, cofibrations $:=\mathrm{Cof}_{T}$ and fibrations $:=\mathrm{Fib}_{T}$ define a proper cofibrantly generated model structure on $\mathcal{P}_{\text {os }}$. The sets $\mathcal{I}$ and $\mathcal{J}$ are sets of generating cofibrations and trivial cofibrations respectively. Moreover, the adjunction pos: $\mathcal{C}$ at $\rightleftarrows \mathcal{P}$ os $: i$ is a Quillen equivalence.

Proof. The only non-trivial part of the first claim is to show that the factorisations exist in $\mathcal{P}$ os. By Proposition 2.4(b), the morphisms in $\mathcal{I}$ and $\mathcal{J}$ are Dwyer morphisms in $\mathcal{P}$ os. By Lemma 2.5, the factorisations in $\mathcal{C}$ at given by the small-object argument applied to a morphism between posets stay inside $\mathcal{P}$ os. It follows that there is a model structure on $\mathcal{P}_{\text {os }}$ as claimed, and that $\mathcal{I}$ and $\mathcal{J}$ are sets of generating cofibrations and trivial cofibrations respectively.

The model structure on $\mathcal{P}$ os is left proper because Thomason's model structure is left proper and Lemma 2.5. It is right proper because $\mathcal{C}$ at is right proper and $i: \mathcal{P}$ os $\rightarrow \mathcal{C}$ at preserves pullbacks.

For the last claim, it suffices to recall that every cofibrant object in $\mathcal{C}$ at is a poset [28, Proposition 5.7]. Every cofibration $\varnothing \rightarrow C$ is a retract of an $\mathcal{I}$-cellular morphism $\varnothing \rightarrow C^{\prime}$. By Lemma 2.5, if $\varnothing \rightarrow C^{\prime}$ is an $\mathcal{I}$-cellular morphism, then $C^{\prime}$ is a poset. Hence $C$ is also a poset and the result follows.

Remark 2.7. The last theorem may be seen as an instance of the following more general situation: let $(\mathcal{M}, W$, Cof, Fib) be a cofibrantly generated model category with $I$ and $J$ sets of generating cofibrations and trivial cofibrations respectively. Suppose $\mathcal{C}$ is a full subcategory which has all small limits and colimits. Then $\left(\mathcal{C}, W_{\mid \mathcal{C}}, \operatorname{Cof}_{\mid \mathcal{C}}, \operatorname{Fib}_{\mid \mathcal{C}}\right)$ is a model category if for every morphism $i: X \rightarrow Y$ in $\mathcal{M}$ that is either $I$-cellular or 
$J$-cellular and $X \in \mathcal{C}$, then $i$ is in $\mathcal{C}$. Furthermore, if $I$ and $J$ are in $\mathcal{C}$ and they satisfy the required smallness condition in $\mathcal{C}$, then $I$ and $J$ are sets of generating cofibrations and trivial cofibrations respectively for the induced model structure on $\mathcal{C}$.

\section{More about cofibrations in $\mathcal{C}$ at and $\mathcal{P}$ os}

An unsatisfactory feature of Thomason's model category is that the definition of cofibrations does not carry a very intuitive meaning, besides perhaps being pseudoDwyer morphisms. The introduction of $S d^{2}$ and $\mathrm{Ex}^{2}$ makes the cofibrations less apparent and more difficult to describe explicitly. In this section, we will show that any set of pseudo-Dwyer morphisms that contains $\mathcal{I}$ is equally good for generating a class of cofibrations for a proper model structure on $\mathcal{C}$ at or $\mathcal{P}$ os.

Let us first recall some notation and terminology about cofibrant generation in a cocomplete category $\mathcal{C}$. A class of morphisms $S$ in $\mathcal{C}$ is called cofibrantly closed if it is closed under retracts, pushouts and transfinite compositions. The cofibrant closure $\operatorname{Cof}_{\mathcal{C}}(S)$ of a class of morphisms $S$ in $\mathcal{C}$ is the smallest cofibrantly closed class of morphisms that contains $S$. A cofibrantly closed class $S$ is called cofibrantly generated if there exists a set of morphisms $I$ such that $S=\operatorname{Cof}_{\mathcal{C}}(I)$.

The model categories $\mathcal{C} a t$ and $\mathcal{P}$ os, besides being cofibrantly generated, are also combinatorial because $\mathcal{C}$ at and $\mathcal{P}$ os are both locally (finitely) presentable.

Definition 3.1 (J.H. Smith). A model category $\mathcal{M}$ is called combinatorial if it is cofibrantly generated and its underlying category is locally presentable.

For background in the theory of locally presentable categories, the reader should consult the excellent monograph $\mathbf{1}$. Combinatorial model categories have many useful properties and they have been studied in $[\mathbf{3}, \mathbf{8}, \mathbf{2 2}$. We are going to need the following important result.

Theorem 3.2. Let $\mathcal{C}$ be a locally presentable category, $W$ a subcategory of $\mathcal{C}$ and $I$ a set of morphisms. Then the classes of morphisms $W, \operatorname{Cof}(I)$ and $(\operatorname{Cof}(I) \cap W)-\operatorname{inj}$ define classes of weak equivalences, cofibrations and fibrations for a cofibrantly generated model structure on $\mathcal{C}$ if and only if the following conditions are satisfied:

(i) $W$ satisfies the two-out-of-three property;

(ii) $I-\operatorname{inj} \subseteq W$;

(iii) $\operatorname{Cof}(I) \cap W$ is closed under transfinite compositions and pushouts;

(iv) $W$ is accessible and accessibly embedded in $\mathcal{C}^{\rightarrow}$.

Proof. By [1, Theorem 2.53], every accessible, accessibly embedded subcategory of a locally presentable category is cone-reflective, hence it satisfies the solution set condition at every morphism. Moreover, it is closed under retracts [3, Proposition 1.19]. Therefore the sufficiency of the conditions follows from J.H. Smith's theorem [3, Theorem 1.7]. The necessity of (i), (ii) and (iii) is obvious. The necessity of (iv) is proved in [15, Corollary A.2.6.6], [22, Theorem 4.1].

Theorem 3.2 has an interesting application to the study of the cofibrations of a combinatorial model category. Beke [4] used a similar method to show the existence 
of countably many different choices of cofibrations for a model structure on $\mathcal{S}$ Set with the usual class of weak equivalences. In this section, we will apply it in the case of the model categories $\mathcal{C}$ at and $\mathcal{P}$ os and the cofibrantly closed class of pseudo-Dwyer morphisms to show that there is a proper class of different choices of cofibrations.

Definition 3.3. A set $S$ of pseudo-Dwyer morphisms in $\mathcal{C}$ at (resp. Pos) is called adequate if $S$-inj $\subseteq \mathcal{W}_{\mathcal{C} a t}$ (resp. $S$-inj $\left.\subseteq \mathcal{W}_{\mathcal{P} \text { os }}\right)$.

For example, the set $\mathcal{I}$ of Theorem 2.2 is adequate. Note that there are formal cofibrations that are not pseudo-Dwyer morphisms. For example, for every pseudo-Dwyer morphism $i: A \rightarrow B$, the opposite functor $i^{\mathrm{op}}: A^{\mathrm{op}} \rightarrow B^{\mathrm{op}}$ is a formal cofibration, but not a pseudo-Dwyer morphism.

Proposition 3.4. Let $S$ be an adequate set of pseudo-Dwyer morphisms in $\mathcal{C}$ at (resp. $\mathcal{P}$ os). Then there is a left proper combinatorial model category $\mathcal{C} t_{S}$ (resp. $\mathcal{P}_{\text {os }}$ ) where:

(a) the underlying category is $\mathcal{C}$ at (resp. Pos),

(b) the class of cofibrations is $\operatorname{Cof}_{\mathcal{C} a t}(S)$ (resp. $\operatorname{Cof}_{\mathcal{P} o s}(S)$ ), and

(c) the class of weak equivalences is $\mathcal{W}_{\mathcal{C} \text { at }}$ (resp. $\left.\mathcal{W}_{\mathcal{P} o s}\right)$.

Moreover, for every two adequate sets $S \subset S^{\prime}$, the identity functor $\mathcal{C}_{S_{S}} \rightarrow \mathcal{C}$ at ${ }_{S^{\prime}}$ (resp. $\left.\mathcal{P o s}_{S} \rightarrow \mathcal{P o s}_{S^{\prime}}\right)$ is a left Quillen equivalence.

Proof. It suffices to verify the conditions of Theorem 3.2; (i) is obvious and (ii) holds by hypothesis. For (iii), note that the class $\operatorname{Cof}(S) \cap \mathcal{W}_{\mathcal{C} a t}$ is closed under transfinite compositions since $\operatorname{Cof}(S)$ is closed under transfinite compositions and it is well-known that a transfinite composition of trivial cofibrations in $\mathcal{S} \mathcal{S}$ et is again a weak homotopy equivalence. Also, since every pseudo-Dwyer morphism is a formal cofibration, the class $\operatorname{Cof}(S) \cap \mathcal{W}_{\mathcal{C} \text { at }}$ is closed under pushouts. Condition (iv) holds because, by Theorem 2.2 and $3.2, \mathcal{W}_{\mathcal{C} a t}$ is accessible and accessibly embedded in $\mathcal{C} a t \rightarrow$. The same argument applies to $\mathcal{P}$ os.

The model structure is left proper for the same reason that the model structure of Theorem 2.2 (resp. Theorem 2.6) is left proper. The last claim is self-evident.

Let $\mu$ be a regular cardinal, i.e., an infinite cardinal which is not a sum of a smaller number of smaller cardinals. Consider the set $\mathcal{I}(\mu)$ of all pseudo-Dwyer morphisms between $\mu$-presentable objects in $\mathcal{C}$ at (resp. $\mathcal{P}$ os). Note that a small category whose set of morphisms has cardinality less than $\mu$ is $\mu$-presentable. The converse is true for posets, but not for small categories in general (e.g., a finitely presentable infinite group is a finitely presentable object in $\mathcal{C}$ at).

Theorem 3.5. The set $\mathcal{I}(\mu)$ is adequate. The combinatorial model category $\mathcal{C} a t_{\mathcal{I}(\mu)}$ (resp. $\left.\mathcal{P o s}_{\mathcal{I}(\mu)}\right)$ is proper and every $\mu$-presentable object is cofibrant. Moreover, for every $\mu<\mu^{\prime}$, the model categories $\mathcal{C} a t_{\mathcal{I}(\mu)}$ and $\mathcal{C} a t_{\mathcal{I}\left(\mu^{\prime}\right)}\left(\right.$ resp. $\mathcal{P}_{\text {os }}{ }_{\mathcal{I}(\mu)}$ and $\left.\mathcal{P}_{o s_{\mathcal{I}\left(\mu^{\prime}\right)}}\right)$ are different.

Proof. Thomason's set of generating cofibrations $\mathcal{I}$ is a set of Dwyer morphisms between finite posets; hence $\mathcal{I} \subseteq \mathcal{I}(\mu)$. Therefore we have that $\mathcal{I}(\mu)-$ inj $\subseteq \mathcal{I}-$ inj $\subseteq$ $\mathcal{W}_{\mathcal{C} a t}$; hence $\mathcal{I}(\mu)$ is adequate. 
By Proposition 3.4, there is a left proper combinatorial model category $\mathcal{C}_{\mathcal{I}(\mu)}$ (resp. $\left.\mathcal{P}_{o s_{\mathcal{I}(\mu)}}\right)$. It is also right proper because every fibration in $\mathcal{C a t}_{\mathcal{I}(\mu)}$ is also a Thomason fibration. Note that the unique morphism $\varnothing \rightarrow C$ is a pseudo-Dwyer morphism for all $C$; hence every $\mu$-presentable object $C$ is cofibrant in $\mathcal{C a t}_{\mathcal{I}(\mu)}$.

For the second claim, note first that Lemma 2.5 implies that $\operatorname{Cof}_{\mathcal{P}_{\text {os }}}(\mathcal{I}(\mu)) \subseteq$ $\operatorname{Cof}_{\mathcal{C} a t}(\mathcal{I}(\mu))$ for every $\mu$. We claim that for every regular cardinal $\mu$, there is a poset $P_{\mu}$ with cardinality $\mu$ that is not cofibrant in $\mathcal{C} a t_{\mathcal{I}(\mu)}$. Then it will follow that the model categories are different because every poset of cardinality less than $\mu^{\prime}$ is $\mu^{\prime}$-presentable in $\mathcal{C}$ at and therefore $P_{\mu}$ is cofibrant in both $\mathcal{C} a t_{\mathcal{I}\left(\mu^{\prime}\right)}$ and $\mathcal{P}_{\text {os }}{ }_{\mathcal{I}\left(\mu^{\prime}\right)}$.

Recall that a cardinal is an ordinal with the property that it is the cardinality of a set. Let $P_{\mu}$ be the poset with the reverse order of $\mu$. Suppose that $P_{\mu}$ is cofibrant in $\operatorname{Cat}_{\mathcal{I}(\mu)}$. This means that it is a retract of an $\mathcal{I}(\mu)$-cell $\tilde{P}_{\mu}$ and let $r: \tilde{P}_{\mu} \rightarrow P_{\mu}$ denote the retraction. The object $\tilde{P}_{\mu}$ can be presented by a sequence of pseudo-Dwyer inclusions

$$
\varnothing=Q_{0} \subsetneq Q_{1} \subseteq Q_{2} \subseteq \cdots \subset Q_{\lambda} \subseteq \cdots \subseteq \tilde{P}_{\mu}
$$

where

(i) every $Q_{\alpha} \rightarrow Q_{\alpha+1}$ is a pushout by a morphism in $\mathcal{I}(\mu)$, and

(ii) for every limit ordinal $\lambda, Q_{\lambda}=\operatorname{colim}_{\alpha<\lambda} Q_{\alpha}$.

The cardinality of $Q_{1}$ is less than $\mu$ and therefore $Q_{1} \neq \tilde{P}_{\mu}$. The pseudo-Dwyer morphism $Q_{1} \rightarrow \tilde{P}_{\mu}$ restricts to a pseudo-Dwyer morphism $r\left(Q_{1}\right) \rightarrow P_{\mu}$ because pseudoDwyer morphisms are closed under retracts by Proposition 2.4(a). Moreover, the poset $r\left(Q_{1}\right)$ has cardinality less than $\mu$. But this leads to a contradiction because every sieve of $P_{\mu}$ is a cofinal sequence in $\mu$ and therefore its cardinality is $\mu$, since $\mu$ is regular.

Remark 3.6. Note that the model category $\mathcal{C} a t_{\mathcal{I}\left(\aleph_{0}\right)}$ is different from Thomason's (see Theorem 2.2) because every finite category is cofibrant in the former, but not in the latter. We do not know if the analogous statement is true for $\mathcal{P}$ os.

Corollary 3.7. The class of pseudo-Dwyer morphisms in $\mathcal{C}$ at (resp. Pos) is not cofibrantly generated.

Proof. Every set of pseudo-Dwyer morphisms is a subset of $\mathcal{I}(\mu)$ for some regular cardinal $\mu$. Therefore, by Theorem 3.5 , it cannot generate the whole class of pseudoDwyer morphisms.

Both categories $\mathcal{C}$ at and $\mathcal{P}$ os are cartesian closed. For every $C$ and $D$ in $\mathcal{C}$ at, there is a natural isomorphism $\operatorname{Fun}(C \times D, E) \cong \operatorname{Fun}(C, \operatorname{Fun}(D, E))$, where $\operatorname{Fun}(C, D)$ denotes the category of functors and natural transformations. Also, note there is a natural isomorphism $N \operatorname{Fun}(C, D) \cong \operatorname{Map}(N C, N D)$.

It is natural to ask whether any of the model structures is compatible with the closed monoidal pairing given by the product. It turns out that this is not true; i.e., none of the model categories above is a monoidal model category in the sense of $\mathbf{1 2}$, Definition 4.2.6]. This can be seen by the following simple example. Consider the 
standard inclusion:

$$
[0] \hookrightarrow[1]
$$

This is a Thomason cofibration because it is a retract of the inclusion

$$
[0] \rightarrow \operatorname{catSd} d^{2}\left(\Delta_{1}\right) .
$$

For the claim, it suffices to show that the map

$$
j:[0] \times[1] \cup_{[0] \times[0]}[1] \times[0] \rightarrow[1] \times[1]
$$

is not a Dwyer morphism. Diagrammatically, the map $j$ is the inclusion of the white dots in the following diagram:

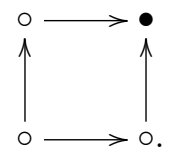

The cosieve generated by the image of the inclusion is clearly the whole poset $[1] \times[1]$, but there can be no retraction because there is nowhere to send the element $(1,1)$. This means that $j$ is not a Dwyer morphism.

However, it is easy to see that for every small category $C$ and pseudo-Dwyer morphism $i: D \rightarrow D^{\prime}$, the map $1 \times i: C \times D \rightarrow C \times D^{\prime}$ is again a pseudo-Dwyer morphism. Hence for every $\mu$-presentable $C$, the functor $C \times-: \mathcal{C}_{t_{\mathcal{I}(\mu)}} \rightarrow \mathcal{C} a t_{\mathcal{I}(\mu)}$ is a left Quillen functor.

\section{Posets and $A$-spaces}

The category $\mathcal{P}_{\text {os }}$ of posets embeds in $\mathcal{T}$ op as the full subcategory of Alexandroff $T_{0}$-spaces.

Definition 4.1. A topological space $X$ is called an Alexandroff $T_{0}$-space $(A$-space) if it is $T_{0}$ and every intersection of open sets in $X$ is open.

For example, every $T_{0}$-space with finitely many points is an $A$-space. Let $\mathcal{A}$ denote the category of $A$-spaces and continuous maps.

Proposition 4.2. There is an isomorphism of categories $\mathcal{T}: \mathcal{P}$ os $\rightleftarrows \mathcal{A}: \mathcal{P}$.

Proof. The assertion is well-known. We recall the proof in order to fix some notation. The functor $\mathcal{T}: \mathcal{P o s} \rightarrow \mathcal{A}$ is defined as follows: for a poset $P, \mathcal{T}(P)$ is the set ob $P$ of objects of $P$ with the topology generated by a basis with sets $U_{a}=\{b: b \geqslant a\}$ for all $a \in \operatorname{ob} P$. The fact that the topology is $T_{0}$ follows from the antisymmetry of the order. It is an $A$-space because any intersection of basic open sets is also a union of basic open sets; i.e., for every $\sigma \subset P$,

$$
U_{\sigma}:=\bigcap_{a \in \sigma} U_{a}=\bigcup_{b \in U_{\sigma}} U_{b}
$$

Moreover, every order-preserving map $f: P \rightarrow P^{\prime}$ defines a continuous map

$$
\mathcal{T}(f): \mathcal{T}(P) \rightarrow \mathcal{T}\left(P^{\prime}\right) .
$$

An inverse functor $\mathcal{P}$ is defined as follows: for an $A$-space $X$, we let $\mathcal{P}(X)$ be the set $X$ with an ordering defined so that $x \geqslant y$ if every open set that contains $y$ also 
contains $x$. The relation is clearly reflexive and transitive. It is antisymmetric because the topology of $X$ is $T_{0}$.

Let $\mathcal{T} o p_{0}$ denote the full subcategory of $\mathcal{T} o p$ that consists of $T_{0}$-spaces.

\section{Proposition 4.3.}

(a) The inclusion $\mathcal{T}$ op $0 \rightarrow \mathcal{T}$ op has a left adjoint.

(b) The inclusion $\mathcal{A} \rightarrow \mathcal{T}$ op $_{0}$ has a right adjoint.

Proof of (a). Let $X \in \mathcal{T}$ op. Define an equivalence relation on the points of $X: x \sim x^{\prime}$ if $x$ and $x^{\prime}$ have exactly the same open neighborhoods. Then taking the quotient $\mathcal{K}(X)=$ $X / \sim$ defines a functor $\mathcal{K}: \mathcal{T} o p \rightarrow \mathcal{T} o p_{0}$ which is the left adjoint to the inclusion functor. The space $\mathcal{K}(X)$ is known as the Kolmogoroff quotient of $X$.

Proof of $(b)$. For every $X \in \mathcal{T} o p_{0}$, let $\Lambda_{\infty}(X)$ denote the $A$-space with underlying set $X$ and whose open sets are unions of arbitrary intersections of open sets in $X$. Then $\Lambda_{\infty}: \mathcal{T} o p_{0} \rightarrow \mathcal{A}$ defines a functor and it is right adjoint to the inclusion functor.

Remark 4.4. The inclusion functor $i: \mathcal{A} \rightarrow \mathcal{T}$ op does not admit a right adjoint. This can be verified by the fact that $T_{0}$-spaces are not closed under pushout in $\mathcal{T}$ op in general. However, they are closed under pushouts along Dwyer morphisms (compare with Lemma 2.5 ).

The class of weak homotopy equivalences $\mathcal{W}_{\mathcal{A}}$ in $\mathcal{A}$ is a natural candidate class to consider in order to define a homotopy theory in $\mathcal{A}$. McCord [18 discovered a surprising connection between the homotopy theory of $A$-spaces and simplicial complexes. Recall that the classifying space functor $B: \mathcal{C} a t \rightarrow \mathcal{T}$ op is the composition of the nerve functor followed by the geometric realisation functor $|\cdot|: \mathcal{S} \mathcal{S}$ et $\rightarrow \mathcal{T}$ op. If $C$ is a poset, then $B C$ is isomorphic with the geometric simplicial complex whose poset of simplices is the poset of the chains in $C$ ordered by inclusion.

Theorem 4.5 (McCord 18). For every A-space $X$, there is a natural weak homotopy equivalence $\psi_{X}: B \mathcal{P}(X) \rightarrow X$. Therefore $\mathcal{W}_{\mathcal{A}}=\mathcal{W}_{\mathcal{P} \text { os }}$.

Proof. We only reproduce a sketch of the proof. For the details, see [18 (also [16 for a nice survey). Every point $x \in B \mathcal{P}(X)$ is an interior point of a simplex spanned by a finite totally ordered subset $\left\{x_{1}<x_{2}<\cdots<x_{n}\right\} \subset X$. Define the map $\psi$ by $\psi_{X}(x)=$ $x_{n}$. It is continuous: for $V$ open in $X, \psi_{X}^{-1}(V)=\cup\{\operatorname{star}(v): v \in V\}$. Also, $\psi$ is clearly functorial in $X$. The restriction $\psi_{X}^{-1}\left(U_{x}\right) \rightarrow U_{x}$ is a weak homotopy equivalence, since both spaces are contractible (they both look like "cones"). Since $\left\{U_{x}\right\}_{x \in X}$ form an (minimal) open covering for $X$, it follows that $\psi_{X}$ is a weak homotopy equivalence.

Using Proposition 4.2 as a dictionary, one can also describe the Dwyer morphisms in the category $\mathcal{A}$ as something analogous to the closed $N D R$-pairs in $\mathcal{T}$ op. In the non-Hausdorff world of $A$-spaces, the role of the unit interval is given to the Sierpiński space $S$, i.e., the $A$-space associated with the poset [1] $=\{0<1\}$. Then the description of the Dwyer morphisms that is given in the following proposition is comparable with the description of the closed (Hurewicz) cofibrations as closed NDR-pairs; see 26 , Theorem 2]. 


\section{Proposition 4.6.}

(a) Let $f: P \rightarrow Q$ be a morphism in $\mathcal{P}$ os. Then the following are equivalent:

(i) $f$ is a sieve,

(ii) there is a morphism $\chi: Q \rightarrow S$ with $\chi^{-1}(0)=P$, and

(iii) the map $\mathcal{T}(f): \mathcal{T}(P) \rightarrow \mathcal{T}(Q)$ is a closed inclusion of spaces.

(b) A morphism $f: P \rightarrow Q$ in $\mathcal{P}$ os is a Dwyer morphism if and only if $\mathcal{T}(f)$ is a closed inclusion and there is an open neighborhood $U$ of $\mathcal{T}(P)$ in $\mathcal{T}(Q)$ and a "Sierpiński homotopy" $H: U \times S \rightarrow \mathcal{T}(Q)$ with $H(u, 1)=u$ for all $u \in U$, $H(u, 0) \in \mathcal{T}(P)$ and $H(p, t)=p$ for all $(p, t) \in \mathcal{T}(P) \times S$.

Proof of (a). The proof of (a) is obvious.

Proof of $(b)$. Open neighborhoods of $\mathcal{T}(Q)$ correspond exactly to the cosieves of $Q$. A Sierpiński homotopy $H$ gives rise to a retraction $r: \mathcal{P}(U) \rightarrow P, u \mapsto H(u, 0)$, together with a natural transformation $r \rightarrow 1$ whose component at $u \in \mathcal{P}(U)$ is given by $H(u, 0<1)$, and vice-versa.

Therefore we can rephrase Theorem 3.5 for $\mu=\aleph_{0}$ by saying that there is a proper cofibrantly generated model structure on $\mathcal{A}$, where the class of weak equivalences is $\mathcal{W}_{\mathcal{A}}$ and the class of cofibrations is generated by the set of maps between finite $A$-spaces that satisfy the conditions of Proposition 4.6(b). There is a zig-zag of right Quillen equivalences from $\mathcal{A}$ to $\mathcal{T}$ op:

$$
\mathcal{A} \stackrel{\cong}{\longrightarrow} \mathcal{P} \text { os } \stackrel{i}{\longrightarrow} \mathcal{C} a t \stackrel{\mathrm{Ex}^{2} N}{\longrightarrow} \mathcal{S} \text { Set } \stackrel{S_{*}}{\longleftarrow} \mathcal{T} \text { op },
$$

and note that the inclusion $i: \mathcal{A} \rightarrow \mathcal{T}$ op induces an equivalence between the homotopy categories by Theorem 4.5. Note also that $\mathcal{A}$ is a subcategory of $\mathcal{T}$ op that is locally presentable.

Remark 4.7. Strøm [27] proved that there is a model structure on $\mathcal{T}$ op, where the cofibrations are the closed (Hurewicz) cofibrations, the weak equivalences are the homotopy equivalences and the fibrations are the Hurewicz fibrations. It was expected that this model structure is not cofibrantly generated. In view of Proposition 4.6(a), the arguments in the proof of Theorem 3.5 can be seen to confirm this. For every set of closed cofibrations $I$ in $\mathcal{T} o p$, there is a regular cardinal $\mu$ greater than the cardinalities of all the spaces that appear in the set. It can then be argued, similarly to the proof of Theorem 3.5, that the map $\varnothing \rightarrow \mathcal{T}\left(P_{\mu}\right)$ is not in the cofibrant closure of $I$. But every space is cofibrant in Strøm's model structure, so it cannot be cofibrantly generated.

There is a stronger notion of weak equivalence in $\mathcal{P}$ os (resp. $\mathcal{C}$ at) that arises from letting the natural transformations play the role of homotopies. More precisely, two morphisms $f, g: C \rightarrow D$ in $\mathcal{C}$ at are called strongly homotopic, denoted by $f \simeq g$, if there is a zig-zag of natural transformations that connects them. The relation of homotopy clearly defines an equivalence relation on the morphism sets of $\mathcal{C}$ at and the composition of functors respects it. A morphism $f: C \rightarrow D$ is a strong homotopy equivalence if there is a morphism $g: D \rightarrow C$ such that $f g \simeq 1_{D}$ and $g f \simeq 1_{C}$. The quotient category $\mathcal{C} a t / \simeq$ is the same as the localisation of $\mathcal{C}$ at at the strong homotopy equivalences. The class of strong homotopy equivalences in $\mathcal{C}$ at has been studied 
by Minian [19] who showed that it supports a $\Lambda$-cofibration structure with natural cylinders given by the zig-zag diagrams.

Proposition 4.8. Every morphism $f: P \rightarrow Q$ in $\mathcal{P}$ os (resp. Cat) has a functorial factorisation $f=p i$, where $i$ a Dwyer morphism and $p$ is a strong homotopy equivalence.

Proof. This follows from the fact that $\mathcal{C}$ at is a $\Lambda$-cofibration category [19. We give the construction of the factorisations in $\mathcal{P}$ os in order to present a choice of cylinder objects. Let $f: P \rightarrow Q$ be a map in $\mathcal{P}$ os. Let $Z_{2}$ denote the following zig-zag poset:

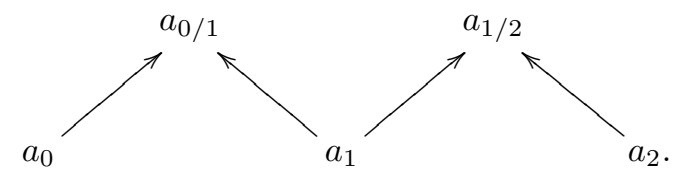

Note that this is just $\operatorname{catSd}^{2}\left(\Delta_{1}\right)$. The inclusion $P \times\left\{a_{0}\right\} \amalg P \times\left\{a_{2}\right\} \rightarrow P \times Z_{2}$ is a Dwyer morphism. Moreover, $P \times Z_{2}$ defines a cylinder object in $\mathcal{P}_{\mathcal{I}} s_{\mathcal{I}(\mu)}$ for every poset $P$ with cardinality less than $\mu$. Define the "mapping-cylinder" $M_{f}$ by the pushout diagram

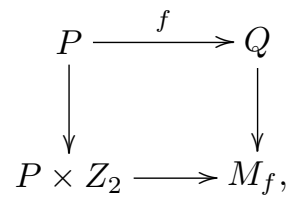

analogously to the mapping-cylinder construction in the category of topological spaces. Explicitly, the poset $M_{f}$ has the underlying set

$$
P \times\left\{a_{0}, a_{0 / 1}, a_{1}, a_{1 / 2}\right\} \cup Q \times\left\{a_{2}\right\},
$$

and the partial order is defined by

1. $(p, x) \leqslant\left(p^{\prime}, y\right)$ if $p \leqslant p^{\prime}$ in $P$ and $x \leqslant y$ in $\left\{a_{0}, a_{0 / 1}, a_{1}, a_{1 / 2}\right\}$,

2. $\left(f(p), a_{2}\right) \leqslant\left(p^{\prime}, a_{1 / 2}\right)$ if $p \leqslant p^{\prime}$ in $P$,

3. $\left(q, a_{2}\right) \leqslant\left(q^{\prime}, a_{2}\right)$ if $q \leqslant q^{\prime}$ in $Q$.

The inclusion $i: P \rightarrow M_{f}$, defined by $p \mapsto\left(p, a_{0}\right)$, is a Dwyer morphism. There is also a canonical morphism $p: M_{f} \rightarrow Q$ defined by

1. $f$ on $P \times\{x\}$, for $x \neq a_{2}$, and

2. the identity on $Q \times\left\{a_{2}\right\}$

so that $f=p i$. The map $p: M_{f} \rightarrow Q$ has a homotopy inverse $s: Q \rightarrow M_{f}$ defined by $q \mapsto\left(q, a_{2}\right)$. The construction is clearly functorial in $f$. A similar construction gives the analogous factorisations in $\mathcal{C}$ at.

Remark 4.9. It is possible to define "higher" dimensional cylinders in $\mathcal{C}$ at by using the simplicial structure of the model category $\mathcal{S} S$ et together with the left Quillen equivalence $c a t S d^{2}: \mathcal{S S e t} \rightarrow \mathcal{C}$ at. For every small category $C$, the cosimplicial object cat $S d^{2}(N C \times \Delta$. $)$ is a cosimplicial resolution of $C$ with respect to Thomason's model structure on $\mathcal{C}$ at. For the theory of cosimplicial resolutions and their application to homotopy colimits, see [1]. 
Since we can identify the category of posets with a category of topological spaces, we can also consider the class of topological homotopy equivalences, that is: morphisms $f: P \rightarrow Q$ between posets that define a homotopy equivalence between the associated $A$-spaces. It turns out that the two concepts of strong and topological homotopy equivalence coincide for finite posets.

Proposition 4.10. A morphism $f: P \rightarrow Q$ between finite posets is a strong homotopy equivalence if and only if it is a topological homotopy equivalence.

Proof. A strong homotopy equivalence clearly induces a topological homotopy equivalence. For the converse, it suffices to show that every two homotopic maps $f, g: \mathcal{T}(P) \rightarrow \mathcal{T}(Q)$ can be connected by a zig-zag of natural transformations. For this we need to look closely at the space $\operatorname{Map}(\mathcal{T}(P), \mathcal{T}(Q))$ with the compact-open topology. Since $\mathcal{T}(P)$ is finite (and therefore locally compact), it turns out that $\operatorname{Map}(\mathcal{T}(P), \mathcal{T}(Q))$ is an $A$-space and its topology is the same as that associated with the poset Fun $(P, Q)$; see [14, 17. Moreover, a function $H: \mathcal{T}(P) \times I \rightarrow \mathcal{T}(Q)$ is continuous if and only if the adjoint path $I \rightarrow \operatorname{Map}(\mathcal{T}(P), \mathcal{T}(Q))$ is continuous. Two elements of an $A$-space are connected by a path if and only if they can be connected by a zig-zag in the associated poset (this follows also from Theorem 4.5). Hence the proposition follows.

Remark 4.11. The last proposition is not true for an arbitrary infinite poset. An example is the infinite poset $\mathcal{N}$, known as the Khalimsky half-line, whose objects are the non-negative integers, and the order is defined by $m<n$ if $m$ is even and $|n-m|=1$. This looks like an infinite zig-zag diagram. It can be shown that it is topologically contractible, but not strongly homotopy equivalent to a point (see [19, Example 2.10] and [19 Remark 2.11]). At the heart of the comparison between strong and topological homotopy equivalence is the delicate point-set topological fact that the space of maps between $A$-spaces with the compact-open topology is not an $A$-space in general; see [14].

The strong homotopy types of finite posets (or finite $T_{0}$-spaces) were completely classified by Stong [25] in terms of a simple combinatorial property (see [17] for a nice survey). Given a poset $P$, an element $p \in P$ is called an upbeat (resp. downbeat) point $^{2}$ if the set of all elements strictly greater (resp. lower) than $p$ has a minimum (resp. maximum); i.e., if there is a $q \in P$ such that $q>p$ (resp. $p>q$ ) and for every $q^{\prime} \in P$ with $q^{\prime}>p$ (resp. $p>q^{\prime}$ ), then $q^{\prime} \geqslant q$ (resp. $\left.q \geqslant q^{\prime}\right)$. The insertion or deletion of upbeat or downbeat points does not change the strong homotopy type of $P$. Let the core of $P$, $\operatorname{core}(P) \subset P$, be a deformation retract of $P$ that is minimal; i.e., it contains neither upbeat nor downbeat elements. One can always reach $\operatorname{core}(P)$ by successively deleting beat points from $P$.

Theorem 4.12 (Stong [25]). Two finite posets $P$ and $Q$ are strongly homotopy equivalent if and only if they have isomorphic cores.

\footnotetext{
${ }^{2}$ This terminology is due to J.P. May [17].
} 


\section{Posets in homotopy theory}

\subsection{What does $B C$ classify?}

Answers to this question have been given in [5, Appendix], 20, 24, 29. They fall into two types: the first one ([5 Appendix], [24] gives a Cech-homotopy style interpretation of the set $[X, B \mathcal{C}]$ in terms of $\mathcal{C}$-cocycles, whereas the second one $(\mathbf{2 0}$, 29]) provides a sheaf-theoretic description in terms of $\mathcal{C}$-principal bundles (or $\mathcal{C}$ sheaves). Each of them generalises the case of $G$-cocycles and $G$-principal bundles respectively, for $G$ a (discrete) group.

Definition 5.1. Let $J$ be a poset and $X$ any topological space. An open covering $\mathcal{U}=\left\{U_{p}\right\}_{p \in J}$ of $X$ is said to be well-indexed by $J$ if $U_{p} \subseteq U_{q}$ whenever $p \geqslant q$ and, for every $x \in X$, the subposet $\left\{p \in J: x \in U_{p}\right\}$ has a greatest element.

In other words, an open covering $\mathcal{U}$ of $X$ well-indexed by $J$ is a functor $\mathcal{U}: J^{\text {op }} \rightarrow$ $\mathcal{O}(X)$ into the poset of open subsets of $X$ such that $X=\bigcup_{p} \mathcal{U}(p)$ and for every $x \in X$, there is $p_{x} \in J$ such that $p_{x} \geqslant q$ whenever $x \in \mathcal{U}(q)$. Let $\operatorname{Cov}_{J}(X)$ denote the set of open coverings of $X$ that are well-indexed by $J$. The definition is natural in $X$ : every map $f: X \rightarrow X^{\prime}$ induces a function $\operatorname{Cov}_{J}(f): \operatorname{Cov}_{J}\left(X^{\prime}\right) \rightarrow \operatorname{Cov}_{J}(X)$, by post-composing with $f^{-1}: \mathcal{O}\left(X^{\prime}\right) \rightarrow \mathcal{O}(X)$, in an obviously functorial way.

Proposition 5.2. For every topological space $X$ and poset $J$, there is a natural bijection between $\mathcal{T} o p(X, \mathcal{T}(J))$ and $\operatorname{Cov}_{J}(X)$.

Proof. For every continuous map $f: X \rightarrow \mathcal{T}(J)$, there is a diagram $\psi_{J}(f): J^{\text {op }} \rightarrow$ $\mathcal{O}(X)$ defined by $\psi_{J}(f)(p)=f^{-1}\left(U_{p}\right)=\{x \in X: f(x) \geqslant p\}$. This defines an open covering well-indexed by $J$ because, for every $x \in X$, the element $f(x)$ is the greatest element of the poset $\left\{p \in J: x \in \psi_{J}(f)(p)\right\}$. On the other hand, given an open covering $\mathcal{U}: J^{\text {op }} \rightarrow \mathcal{O}(X)$ well-indexed by $J$, there is a continuous map $\phi_{J}(\mathcal{U}): X \rightarrow$ $\mathcal{T}(J)$ defined by $\phi_{J}(\mathcal{U})(x)=p_{x}$. The functions $\psi_{J}$ and $\phi_{J}$ are mutual inverses and they induce a bijection between $\operatorname{Cov}_{J}(X)$ and $\mathcal{T} o p(X, \mathcal{T}(J))$. The naturality statement amounts to noticing that for every map $f: X \rightarrow Y, \psi_{J} f^{*}=\operatorname{Cov}_{J}(f) \psi_{J}$, where $f^{*}: \mathcal{T} o p(Y, \mathcal{T}(J)) \rightarrow \mathcal{T} o p(X, \mathcal{T}(J))$ is given by precomposition with $f$.

Definition 5.3. Two open coverings $\mathcal{U}$ and $\mathcal{V}$ of $X$ that are well-indexed by $J$ are called concordant if there is an open covering $\mathcal{W}$ of $X \times[0,1]$ well-indexed by $J$ that restricts to $\mathcal{U}$ and $\mathcal{V}$ at $X \times\{0\}$ and $X \times\{1\}$ respectively.

Concordance generates an equivalence relation on $\operatorname{Cov}_{J}(X)$. For the set of equivalence classes, we will write $\left[\operatorname{Cov}_{J}(X)\right]$. Note that for every $f: X \rightarrow X^{\prime}, \operatorname{Cov}_{J}(f)$ preserves the concordance relation.

Proposition 5.4. For every $\mathrm{CW}$-complex $X$ and poset $J$, there is a natural bijection between $[X, B J]$ and $\left[\operatorname{Cov}_{J}(X)\right]$.

Proof. By Theorem 4.5, there is a natural weak homotopy equivalence $\psi: B J \rightarrow \mathcal{T}(J)$ and therefore a bijection $\psi_{*}:[X, B J] \rightarrow[X, \mathcal{T}(J)]$. Note that, under the bijection of Proposition 5.2, two maps $X \rightrightarrows \mathcal{T}(J)$ are homotopic if and only if the associated open coverings $J^{\mathrm{op}} \rightrightarrows \mathcal{O}(X)$ are concordant. Thus there is a natural bijection as required. 
Remark 5.5. It is not difficult to see that Proposition 5.4 is the same with the classification theorem by Weiss 29 restricted to the case of posets. There is a different, but analogous, classification theorem due to Moerdijk [20 which makes the same claim at least for finite posets (up to a different variance convention).

Corollary 5.6. A map of CW-complexes $f: X \rightarrow Y$ is a homotopy equivalence if and only if $\left[\operatorname{Cov}_{J}(f)\right]:\left[\operatorname{Cov}_{J}(Y)\right] \rightarrow\left[\operatorname{Cov}_{J}(X)\right]$ is a bijection for all posets $J$.

For a small category $C$, let $\operatorname{Simp}(C)$ denote the poset $\operatorname{cat} S d^{2}(N C)$. Then we also have the following more general result.

Corollary 5.7. For every $\mathrm{CW}$-complex $X$ and small category $C$, there is a natural bijection between $[X, B C]$ and $\left[\operatorname{Cov}_{\operatorname{Simp}(C)}(X)\right]$.

Proof. The natural transformation $S d \rightarrow 1_{\mathcal{S S} \text { et }}$ induces a natural weak equivalence $p: \operatorname{Simp}(C) \rightarrow C$. Therefore there is a bijection $p_{*}:[X, B \operatorname{Simp}(\mathcal{C})] \rightarrow[X, B \mathcal{C}]$. Then the result follows from Proposition 5.4 .

\subsection{Posets of coverings and manifolds}

There is a standard way to produce posets from coverings of topological spaces. Every numerable open covering $\mathcal{U}=\left\{U_{\alpha}\right\}_{\alpha \in I}$ of a space $X$ gives rise to two posets, a topological one denoted by $X_{\mathcal{U}}$, and a discrete one denoted by $I_{\mathcal{U}}$. The space of objects of $X_{\mathcal{U}}$ is $\amalg U_{\sigma}$, over all finite subsets $\sigma$ of the index set $I$ such that $U_{\sigma}=\cap_{\alpha \in \sigma} U_{\alpha}$ is non-empty. More precisely, an object is a pair $\left(U_{\sigma}, x\right)$ where $x \in U_{\sigma}$, and there is a unique morphism from $\left(U_{\sigma}, x\right)$ to $\left(U_{\sigma^{\prime}}, y\right)$ if and only if $x=y$ and $\sigma^{\prime} \subset \sigma$. The category $I_{\mathcal{U}}$ is the discretisation of this category: its objects are the finite subsets $\sigma$ of the index set of the covering $\mathcal{U}$ such that $U_{\sigma} \neq \emptyset$ and its morphisms are given by reverse inclusions of sets. If we view the covering as a $I_{\mathcal{U}}$-diagram in $\mathcal{T}_{o p}$, then $X_{\mathcal{U}}$ is exactly the associated transport category, and $B X_{\mathcal{U}}$ is the Bousfield-Kan model for its homotopy colimit.

An open covering $\mathcal{U}$ is called good if all the finite intersections of its members are either contractible or empty. When $X$ is a smooth $n$-manifold, we reserve the notion to mean that all the non-empty finite intersections are diffeomorphic to $\mathbb{R}^{n}$.

Proposition 5.8 (Segal 23 ). Let $X$ be a paracompact space and $\mathcal{U}$ an open covering. Then the canonical map $p_{\mathcal{U}}: B X_{\mathcal{U}} \rightarrow X$ is a homotopy equivalence. If the covering $\mathcal{U}$ is good, then the projection $q_{\mathcal{U}}: B X_{\mathcal{U}} \rightarrow B I_{\mathcal{U}}$ is also a homotopy equivalence.

Let $\mathcal{M}_{n}$ denote the discrete monoid of smooth embeddings $\mathbb{R}^{n} \rightarrow \mathbb{R}^{n}$. A good covering $\mathcal{U}$ of a smooth $n$-dimensional manifold $M$ produces a (non-canonical) diagram $F_{\mathcal{U}}: I_{\mathcal{U}} \rightarrow \mathcal{M}_{n}$. This object separates the locally Euclidean structure of $M$ from its homotopy type. Moreover, it produces an invariant of $M$, in the sense that the homotopy class of the map

$$
M \stackrel{\simeq}{\longrightarrow} B M_{\mathcal{U}} \stackrel{\simeq}{\longrightarrow} B I_{\mathcal{U}} \stackrel{B\left(F_{\mathcal{U}}\right)}{\longrightarrow} B \mathcal{M}_{n}
$$

classifies the concordance class of the tangent bundle of $M$ as a foliated smooth microbundle; see [24]. Here the first two maps are the homotopy equivalences that come from Proposition 5.8. It would be interesting to have a characterisation of the posets $I_{\mathcal{U}}$ and the diagrams $F_{\mathcal{U}}$ that arise this way from a given manifold $M$. 


\subsection{Sheaves on an $A$-space}

Let $X$ be an $A$-space and let $\operatorname{Sh}(X)$ denote the Grothendieck topos of sheaves on $X$.

Proposition 5.9. There is an equivalence of categories between

$$
\operatorname{Sh}(X) \quad \text { and } \widehat{\mathcal{P}(X)^{\mathrm{op}}}:=\operatorname{Fun}(\mathcal{P}(X), \mathcal{S} e t)
$$

Proof. The restriction functor $R: \operatorname{Sh}(X) \rightarrow \widehat{\mathcal{P ( X ) ^ { \mathrm { op } }}}$ that sends a sheaf $F: \mathcal{O}(X)^{\mathrm{op}} \rightarrow$ Set to $R(F): \mathcal{P}(X) \rightarrow \mathcal{S}$ et, $x \mapsto F\left(U_{x}\right)$ (in the notation of Proposition 4.2) is an equivalence of categories because $\left\{U_{x}\right\}_{x \in X}$ is a basis of minimal open sets for the topology of $X$.

In view of Theorem 4.5, the last proposition has the following immediate consequence. It is known that the singular cohomology $H^{*}(X, G)$ of a CW-complex $X$ with coefficients in an abelian group $G$ is isomorphic with the cohomology $H^{*}(\operatorname{Sh}(X), \mathcal{G})$ of the topos of sheaves on $X$ at the abelian sheaf that is constant at $G$. Every CWcomplex is homotopy equivalent to $B J$ for some poset $J$. Moreover, a homotopy equivalence can be chosen canonically up to homotopy, as can be seen by the following zig-zag of natural homotopy equivalences:

$$
B\left(\operatorname{cat} S d^{2}\left(S_{*}(X)\right)\right) \stackrel{\simeq}{\longleftarrow}\left|S d^{2} S_{*}(X)\right| \stackrel{\simeq}{\longrightarrow}\left|S_{*}(X)\right| \stackrel{\simeq}{\longrightarrow} X .
$$

By Theorem 4.5, there is a weak homotopy equivalence $f: X \rightarrow \mathcal{T}(J)$. Since both $X$ and $\mathcal{T}(J)$ have a basis of contractible open sets, it follows that the topoi $\operatorname{Sh}(X)$ and $\operatorname{Sh}(\mathcal{T}(J)$ ) are weakly homotopy equivalent. (For a quick introduction in the homotopy theory of topoi, see [20, I.4].) In particular, there is an isomorphism $H^{*}(\operatorname{Sh}(X), \mathcal{G}) \cong$ $H^{*}(\operatorname{Sh}(\mathcal{T}(J)), \mathcal{G})$. By Proposition 5.9 , there is an equivalence between $\operatorname{Sh}(\mathcal{T}(J))$ and $\mathcal{S} e t^{J}$, hence also an isomorphism $H^{*}(\operatorname{Sh}(\mathcal{T}(J)), \mathcal{G}) \cong H^{*}\left(\mathcal{S e t}^{J}, \mathcal{G}\right)$. In summary, the cohomology of the topos of sheaves on a (finite) CW-complex is isomorphic with the cohomology of the topos of presheaves on a (finite) poset.

\section{References}

[1] J. Adámek and J. Rosický, Locally presentable and accessible categories, London Math. Soc. Lecture Note Series, No. 189, Cambridge University Press, Cambridge, 1994.

[2] J.A. Barmak and E.G. Minian, Simple homotopy types and finite spaces, Adv. Math. 218 (2008), no. 1, 87-104.

[3] T. Beke, Sheafifiable homotopy model categories, Math. Proc. Cambridge Philos. Soc. 129 (2000), no. 3, 447-475.

[4] T. Beke, Fibrations of simplicial sets, Appl. Categor. Struct. 18 (2010), 505516.

[5] R. Bott, Lectures on characteristic classes and foliations [Notes by L. Conlon, with two appendices by J. Stasheff], Lecture Notes in Math. 279, pp. 1-94, Springer-Verlag, New York, 1972. 
[6] D.-C. Cisinski, La classe des morphismes de Dwyer n'est pas stable par retractes, Cahiers Topologie Géom. Différentielle Catég. 40 (1999), no. 3, 227231.

[7] D.-C. Cisinski, Les préfaisceaux comme modéles des types d'homotopie, Astérisque, No. 308, 2006.

[8] D. Dugger, Combinatorial model categories have presentations, Adv. Math. 164 (2001), no. 1, 177-201.

[9] P.G. Goerss and J.F. Jardine, Simplicial homotopy theory, Progress in Math. 174, Birkhäuser Verlag, Basel, 1999.

[10] R. Fritsch and D.M. Latch, Homotopy inverses for nerve, Math. Z. 177 (1981), no. $2,147-179$.

[11] P.S. Hirschhorn, Model categories and their localizations, Mathematical Surveys and Monographs, Vol. 99, Amer. Math. Soc., Providence, RI, 2003.

[12] M. Hovey, Model categories, Mathematical Surveys and Monographs, Vol. 63, Amer. Math. Soc., Providence, RI, 1999.

[13] D.M. Kan, On c.s.s. complexes, Amer. J. Math. 79 (1957), 449-476.

[14] M.J. Kukieła, On homotopy types of Alexandroff spaces, Order 27 (2010), no. $1,9-21$.

[15] J. Lurie, Higher topos theory, Annals of Mathematics Studies, No. 170, Princeton University Press, Princeton, NJ, 2009.

[16] J.P. May, Finite spaces and simplicial complexes, Notes for REU, available at http://www.math.uchicago.edu/ may.

[17] J.P. May, Finite topological spaces, Notes for REU, available at http://www. math.uchicago.edu/ may.

[18] M.C. McCord, Singular homology groups and homotopy groups of finite topological spaces, Duke Math. J. 33 (1966), 465-474.

[19] E.G. Minian, Cat as a $\Lambda$-cofibration category, J. Pure Appl. Algebra 167 (2002), no. 2-3, 301-314.

[20] I. Moerdijk, Classifying spaces and classifying topoi, Lecture Notes in Math. 1616, Springer-Verlag, New York, 1995.

[21] D.G. Quillen, Homotopical algebra, Lecture Notes in Math. 43, Springer-Verlag, New York, 1967.

[22] J. Rosický, On combinatorial model categories, Appl. Categ. Struct. 17 (2009), 303-316.

[23] G. Segal, Classifying spaces and spectral sequences, Inst. Hautes Études Sci. Publ. Math. 34 (1968), 105-112.

[24] G. Segal, Classifying spaces related to foliations, Topology 17 (1978), no. 4, 367-382.

[25] R.E. Stong, Finite topological spaces, Trans. Amer. Math. Soc. 123 (1966), $325-340$.

[26] A. Strøm, Note on cofibrations, Math. Scand. 19 (1966), 11-14. 
[27] A. Strøm, The homotopy category is a homotopy category, Arch. Math. (Basel) 23 (1972), 435-441.

[28] R.W. Thomason, Cat as a closed model category, Cahiers Topologie Géom. Différentielle 21 (1980), no. 3, 305-324.

[29] M. Weiss, What does the classifying space of a category classify?, Homology Homotopy Appl. 7 (2005), no. 1, 185-195 (electronic).

George Raptis graptis@mathematik.uni-osnabrueck.de

Institut für Mathematik, Universität Osnabrück, Albrechtstrasse 28a, 49069 Osnabrück, Germany 